\title{
Two novel gene-specific markers at pik locus facilitate the application of rice blast resistant alleles in breeding
}

\section{Dagang Tian}

Fujian Academy of Agricultural Sciences

\section{Ziqiang Chen}

Fujian Academy of Agricultural Sciences

Yan Lin

Fujian Academy of Agricultural Sciences

\section{Zaijie Chen}

Fujian Academy of Agricultural Sciences

\section{Jiami Luo}

Fujian Academy of Agricultural Sciences

\section{Mo Wang}

Fujian Agriculture and Forestry University

\section{Songbiao Chen}

Minjiang University

\section{Pingsheng Ji Ji}

University of Georgia

Liming Yang ( $\square$ yangliming@njfu.edu.cn )

Nanjing Forestry University https://orcid.org/0000-0002-8826-9711

\section{Zhonghua Wang}

Fujian Agriculture and Forestry University

\section{Feng Wang}

Fujian Academy of Agricultural Sciences

\section{Original article}

Keywords: Rice, Blast disease, Molecular marker, Pik-p, Pi-1, K-type alleles

Posted Date: December 12th, 2019

DOl: https://doi.org/10.21203/rs.2.18858/v1 
License: (c) (i) This work is licensed under a Creative Commons Attribution 4.0 International License. Read Full License 
1 Two novel gene-specific markers at Pik locus facilitate the application of rice

2 blast resistant alleles in breeding

3 Dagang Tian ${ }^{1,2}$, Ziqiang Chen ${ }^{1}$, Yan Lin $^{1}$, Zaijie Chen ${ }^{1}$, Jiami Luo ${ }^{1}$, Mo Wang ${ }^{2}$, Songbiao Chen $^{3}$, Pingsheng Ji ${ }^{5}$,

4 Liming Yang ${ }^{4,5 *}$, Zhonghua Wang ${ }^{2,3 *}$ and Feng Wang ${ }^{1 *}$

5 'Biotechnology Research Institute, Fujian Key Laboratory of Genetic Engineering for

6 Agriculture, Fujian Academy of Agricultural Sciences, Fuzhou 350003, China;

$7 \quad{ }^{2}$ State Key Laboratory of Ecological Pest Control for Fujian and Taiwan Crops, College of Life

8 Science, Fujian Agriculture and Forestry University, Fuzhou, China;

$9{ }^{3}$ Marine and Agricultural Biotechnology Laboratory, Institute of Oceanography, Minjiang 10 University, Fuzhou 350108, China;

$11{ }^{4}$ College of Biology and the Environment, Nanjing Forestry University, Nanjing, 210037, China;

$12 \quad{ }^{5}$ Department of Plant Pathology, University of Georgia, Tifton, GA 31793, USA

$13 *$ Corresponding authors:

14 Liming Yang, College of Biology and the Environment, Nanjing Forestry University, Nanjing,

15 210037, China; E-mail: yangliming@njfu.edu.cn

16 Zhonghua Wang, State Key Laboratory of Ecological Pest Control for Fujian and Taiwan Crops,

17 College of Life Science, Fujian Agriculture and Forestry University, Fuzhou, China; and Marine

18 and Agricultural Biotechnology Laboratory, Institute of Oceanography, Minjiang University,

19 Fuzhou 350108, China;wangzh@,fafu.edu.cn;

20 Feng Wang, Biotechnology Research Institute, Fujian Key Laboratory of Genetic Engineering

21 for Agriculture, Fujian Academy of Agricultural Sciences, Fuzhou 350003, China; wf@fjage.org 


\section{Abstract}

Background: Rice blast disease, caused by Magnaporthe oryzae, is a major constraint for rice production in the world. Introgression of blast-durable resistance genes into high-yielding rice cultivars has been considered an agricultural priority in an effort to control the disease. The blast resistance Pik locus, located on chromosome 11, contains at least six important resistance genes, but these genes have not been widely employed in resistance breeding since existing markers hardly satisfy current breeding needs owing to their limited scope of application..

Results: In the present study, two PCR-based markers, Pikp-Del and Pi1-In, were developed to target the specific InDel (insertion/deletion) of the Pik-p and $P i-1$ genes, respectively. The two markers precisely distinguished Pik-p, Pi-1, and the K-type alleles at the Pik locus, which is a necessary element for functional genes from rice varieties.

Conclusions:Two gene-specific markers of $P i-k p$ and $P i 1$ identified that only several old varieties contain the two genes, nearly half these varieties yet carry the K-type alleles. Therefore, these identified varieties can be new gene sources for developing blast resistant rice. The two newly developed markers should be highly useful for using Pi-kp, Pil and other resistance genes at the Pik locus in marker-assisted selection (MAS) breeding programs.

Keywords: Rice, Blast disease, Molecular marker, Pik-p, Pi-1, K-type alleles 


\section{Background}

43 Rice (Oryza sativa L.) is one of the most important cereal crops in the world that provides a 44 staple food source for over half of the world's population (Khush 2005). One major obstacle encountered in rice production is the frequent widespread occurrence and damage of rice blast, caused by the ascomycete fungus Magnaporthe oryzae (Jeung et al. 2007). Cloning and utilizing resistance genes in rice breeding is an important and effective approach to enhance cultivar resistance in an economical and safe manner (Zeigler et al. 1994). To date, more than 100 resistance $(R)$ genes have been found, and at least 28 of these genes have been successfully cloned (Deng et al. 2017; Li et al. 2019; Ma et al. 2015). Three of which are important clusters et al. 2016; Deng et al. 2017; Wang et al. 2017). One such cluster is the Pik locus on chromosome 11, which has been commonly considered to be the major locus in control of rice blast disease since it contains at least 6 identified blast $R$-genes, including Pi-1 (Hua et al. 2012), Pik-h (Zhai et al. 2014), Pi-k (Zhai et al. 2011), Pik-m (Ashikawa et al. 2008), Pik-s (Wang et al. 2009), and Pik-p (Yuan et al. 2011). Previous studies have shown that many $R$-genes from the Pik locus confer broad-spectrum resistance to a diverse array of $M$. oryzae isolates (Hua et al. 2012). Due to this fact, the Pik locus has the potential to be used in a marker-assisted selection (MAS) breeding program for the improvement of blast resistance in rice.

Gene-specific markers developed from DNA polymorphisms among the alleles are particularly useful for MAS breeding and screening large amounts of germplasm for allelic diversity (Brar and Khush 1997; Kumari et al. 2013; Nawaz et al. 2017; Shomura et al. 2008; Xu et al. 2005). Such markers have been developed for genes including Pit, Pi-2, Pi-9, and Pig-m (Hayashi et al. 2010; Tian et al. 2016; Wang et al. 2019). In the case of the Pik locus, which displays high similarity among $P i-1, P i k-h, P i-k, P i k-m, P i k-s, P i k-p$, and wild rice clusters from the same region (Hua et al. 2012; Zhai et al. 2011), it is difficult to differentiate these genes from 
each other by simple sequence repeat (SSR) markers. Though several derived cleaved amplified polymorphic sequences (dCAPS) have been developed that allow for screening of Pi-1, Pik-p, Pik-m, and Pi-k genes in cultivars (Chen et al.2016; Hua et al. 2012; Zhai et al. 2011), it is inconvenient to apply these markers to large-scale breeding practices for dCAPS markers since they need to be digested with PCR products and restriction endonucleases, which is laborious and time consuming.

The objectives of this study were:1) to analyze polymorphism in the Pik locus and develop Pi-1, Pik-p, and K-type allele marker systems; 2 ) using the newly developed marker systems to identify the presence of two blast $R$-genes in a large collection of 531 rice accessions; and 3) to verify the resistance of representative rice varieties by pathogenicity tests. Development of the gene-specific markers has great potential applicability in MAS breeding programs.

\section{Results}

\section{Functional markers for Pi-1/Pik-p/K-type genotypes}

To determine the genotypes of $P i-1$ and $P i k-p$, an extensive sequence alignment was conducted to analyze the InDel polymorphisms among the sequences of Pi-1, Pi-k, Pik-m, Pik-p, Pik-s, and Pik-m. A conserved 19-bp deletion at the -1015-bp location of Pikp-1, which is located at the promoter region of Pikp-1, and a 10-bp insertion positioned at +6816-bp of Pil-5-terminal were identified (Fig. 1A). To further verify the two identified InDel polymorphisms, two pairs of specific primers, Pikp-Del and Pi1-In, were designed to develop a molecular marker that targeted the regions containing the identified InDel (Table 1). PCR fragments corresponding to the two regions of $P i-1, P i k-p, P i-k, P i k-m, P i k-h, P i k-s$, and non-functional N-type alleles were amplified from rice monogenic lines IRBL1-CL-Pi1, IRBLkp-K60-Pik-p, IRBLk-Ka-Pik, IRBLkm-Ts-Pik-m, IRBLkh-K3-Pik-h, IRBLks-S-Pik-s, and Nipponbare with specific primers 
91 for the Pikp-Del and Pi1-In markers, respectively. Electrophoretic analysis showed that the band-type of Pik-p and Pi-l were different from the others, and the N-type from Nipponbare had invisible PCR product (Fig. 1D). Therefore, the two developed InDel markers could clearly distinguish the genotypes corresponding to the Pi-1 and Pik-p loci from other functional and non-functional alleles.

\section{Assessment of the Pi-1, Pik-p, and K-type alleles in 531 rice varieties}

A total of 531 rice varieties were assessed for the presence of the Pik-p, Pi-1, and K-type alleles using the newly developed markers. The 531 rice accessions consisted of 98 indica restorer lines of hybrid rice, 49 maintainer/sterile lines of hybrid rice, 328 indica conventional cultivars/breeding materials, and 56 japonica conventional cultivars/breeding materials (supplementary Table S1). When using the Pikp-Del marker for screening, 5 out of 531 and 264 out of 531 accessions were separately identified displaying 368-bp and 377-bp PCR fragment, respectively (supplementary Table S2), suggesting that the 5 accessions might contain the Pik-p resistance genes and the 264 varieties had the functional K-type alleles. When using the Pi1-In marker for screening, 8 and 259 accessions produced specific patterns identical to that of the $P i-1$ and K-type alleles, respectively. Taken together, these results suggest that the 259 accessions may contain the other resistance genes except for the 8 that contain $\mathrm{Pi}-1$ and the 5 that have Pik-p.

The rice accessions containing Pik-p, Pil and K-type identified by the Pil-In and Pikp-Del markers were also confirmed with $\mathrm{K}$ - and $\mathrm{N}$ - type specific primer sets, two dCAPS markers of dCAPS-Pi1 and dCAPS-795 (Zhai et al. 2011; Hua et al. 2012). Genotyping results of the those markers showed consistent with the results using the Pikp-Del and Pil-In markers. The 5 accessions were identified to have a Pik-p genotype corresponding to the Pik-p allele (Fig. 2A, Table S2), and PilFNP analysis in the 8 accessions gave a restriction enzyme digestion pattern identical to the $P i-1$ allele (Fig. 2C, Table S2). 


\section{Disease reaction of varieties possessing $P i-1$ and $P$ ik-p genotypes}

117 To investigate whether the $P i-k p, P i-1$, and K-type varieties showed corresponding specific 118 resistance, 25 representative rice varieties with $P i-1, P i k-p$, and K-type alleles were selected and 119 inoculated with seven blast pathogen isolates KJ201,CHNOS60-2-3, CHE86061, FJ86, FJ501-3, 120 CHL768 (AvrPi-1), and CHL2110 (AvrPik-p) (Hua et al. 2012; Pan et al. 2003). The last two 121 isolates were for resistance identification of the corresponding $\mathrm{R}$ genes, and the other 5 isolates 122 were used for resistance comparison of the 25 representative varieties.

As shown in Table 2 and Figure 3, the 4 Pik-p genotype varieties showed high resistance to CHL2110, FJ86, FJ501-3, CHL768, and CHNOS60-2-3, but moderate resistance to CHE86061, which was similar to or even higher than the disease reactions in the Pikp-carrying line IRBLkp-K60 (Table 3). The 8 Pil-type varieties also appeared equivalent resistance to or had stronger resistance than the Pil-carrying near-isogenic line IRBL1-CL.The 6 K-type varieties showed differences in resistance spectrum compared to the Pik-p, Pik-m, Pik-h, or Pi-1 donor lines, suggesting that these varieties likely possessed other blast $R$-genes from the Pik locus.

\section{Discussion}

The Pik locus has been considered to confer broad spectrum blast resistance. It contains at least six resistance genes (Ashikawa et al. 2008; Hua et al. 2012; Kiyosawa 1987; Wang et al. 2009,

134 2010; Zhai et al. 2011). Pik- $p$ contributes to higher resistance responses in many regions in China 135 including Guangdong, Jiangsu, and Sichuan provinces, indicating that this gene has greater 136 applicability in these regions (Wang et al. 2009, 2017). $P i-1$ is regarded as having great potential 137 for achieving broad spectrum and durable-resistance against blast (Hu et al. 2010; Jiang et al. 138 2012; Li et al. 2005; Wang et al. 2017; Yang et al. 2008; Zhang et al. 2010). The other resistance 139 genes at the locus also have different resistance spectra to the blast fungal pathogen (Hua et al. 
2012).

Utilization of molecular markers has made it feasible to test for the presence of different $\mathrm{R}$ genes, which was previously unattainable whenever several $\mathrm{R}$ genes conferred resistance to overlapping spectra of race-resistance in an inoculation test. With the availability of sequence data for functional $R$-genes, gene-specific DNA markers have been developed for several blast R-genes, such as Pit (Hayashi et al. 2010), Pi54 (Ramkumar et al. 2011), Pi-2/Piz-t/Pi-9 (Hua et al. 2015; Tian et al. 2016), and Pita (He et al. 2014) by using bioinformatics strategies. Not only can these gene-specific markers be used for screening for novel alleles in germplasm sets, but they can also be an effective selection tools for multiple allelic pyramid breeding.

Since the Pik locus contains Pik-p, Pik-m, Pik-h, Pi-1, Pi-k, and Pik-s alleles, which confers broad-spectrum and durable-resistance to rice blast disease (Hua et al. 2012), it is of great significance to accelerate its application in breeding practices. The current study developed Pikp-Del and Pil-In markers based on the gene-specific states of Pik-p and Pi-1 genes, respectively. The Pikp-Del marker lies in between Pik-p1(K1) and Pik-p2(K2), only 830-bp and 2,576-bp away from the K1 and K2 marker, respectively. And the Pi1-In marker located at the +6816 bp away from the start position of Pi1-5(Fig.1). All in all, the present study provides a unique opportunity to swiftly screen large scale germplasm resources for the target gene with high accuracy, which is very different from former dCAPS marker method which needed to digest PCR products with restriction endonuclease. So the newly developed markers have the ability to fix alleles in a population with more efficiency, which will be particularly useful in MAS breeding methods.

Additionally, we found that $P i-1$ or Pik-p only exists in several older local or bred varieties, such as RCPL3-2, Ruanmigu and Zaohui62, and few newly-bred varieties contained them, So we can infer that the two genes have not been widely applied in rice breeding. In addition, the 6 representative K-type allelic varieties also showed different resistance responses from the four 
165

166

167

168

169

170

171

172

\section{Conclusions}

174 In this study, we developed two gene-specific markers for Pik-p and Pil, and identified the 175 presence of the two blast $R$-genes in 531 rice varieties based on the two new developed marker.

176 The results revealed that Pi-1 or Pik-p only exists in several older local or bred varieties and 177 nearly half the identified varieties carry the K-type alleles, supporting that the two blast- $R$ genes 178 and the K-type alleles can be important gene sources for breeding of blast resistant in future. 179 Furthermore, the Pikp-Del and Pi1-In markers will be highly useful in MAS breeding for blast 180 resistance.

181

\section{Methods}

183 Materials and Methods

184 Plant material and pathogen isolates

185 The 531 rice varieties were maintained at Fujian Provincial Key Laboratory of Genetic 186 Engineering for Agriculture, Fujian Academy of Agricultural Sciences, Fuzhou, China. Six 
monogenic lines, IRBLkh-K3 (Pik-h), IRBLkm-Ts $(P i k-m)$, IRBLkp-K60 (Pik-p), IRBLk-Ka (Pi-k), IRBLks-F5 (Pik-s) and IRBL1-CL (Pi-1), were developed by International Rice Research Institute (IRRI) (Tsunematsu et al. 2000) and provided by Dr. Di Liu at Chinese Academy of Tropical Agricultural Sciences. Seven Magnaporthe oryzae isolates, KJ201, CHE86061, CHNOS60-2-3, FJ86, PO6-6, CHL2110 and CHL768, were kindly provided by Dr. Qinghua Pan (Laboratory of Plant Resistance and Genetics, South China Agricultural University) and Dr. Guo-Liang Wang (Department of Plant Pathology, Ohio State University). M. oryzae isolates were cultured on oatmeal agar plates in the dark for 7 days at $28^{\circ} \mathrm{C}$ and then grown under light and dark cycles of $12 / 12 \mathrm{~h}$ for 5 days.

\section{Rice blast inoculation assay}

Rice blast inoculation assays were conducted in a greenhouse with an automation system to maintain a suitable temperature around $28^{\circ} \mathrm{C}$ and relative humidity of $>95 \%$. About 2 weeks after sowing, 20 rice seedlings of each of 25 rice varieties were spray-inoculated with spore suspensions of the 7 isolates separately at a concentration of $5 \times 10^{5}$ spores $/ \mathrm{ml}$ until run-off. After inoculation, the cement tank was completely covered with a tent, and the inoculated seedlings were maintained under dark conditions for $24 \mathrm{~h}$. The tent was then removed from the tank, and the seedlings were grown in the greenhouse under high humidity $(>95 \%)$ for an additional week for symptom evaluation. The 0-5 standard scale was used to score disease severity following Campbell et al. (2004). Plants with lesion scores 0, 1 and 2 were considered to be resistant, 3, 4 and 5 were considered as moderately resistant, susceptible and highly susceptible, respectively.

\section{DNA extraction}

Leaf tissues of rice plants were collected for DNA extraction. About $50 \mathrm{mg}$ of leaf samples collected in Eppendorf tubes were frozen in liquid nitrogen and were processed into fine powder with a TissueLyser (Qiagen, Haan, Germany). Rice genomic DNA was extracted from ground 
212 leaf tissue by the Cetyltrimethyl Ammonium Bromide (CTAB) method as described by Murray 213 and Thompson (1980).

\section{Development of allele-specific markers for Pi-1 and Pik-p}

215 Based on the multiple sequence alignment of Pi-1 (HQ606329), Pik-h (HQ662330), Pi-k 216 (HM048900), Pik-m (GU811852), Pik-s (HQ662329), and Pik-p (HM035360), Pikp-Del and

217 Pi1-In markers were designed specifically targeting the InDel polymorphisms of Pik-p and Pi-1 218 genes among Pi-1, Pik-h, Pi-k, Pik-m, Pik-s, and Pik-p. PCR was performed in a $25 \mu 1$ reaction 219 that contained $12.5 \mu \mathrm{l}$ of $2 \mathrm{x}$ mix buffer (Mg2+Plus), $1 \mu \mathrm{l}$ of each primer $(10 \mathrm{mM})$, and $0.2 \mu \mathrm{l}$ 220 Taqase $(5 \mathrm{U} / \mathrm{ml})$. For each reaction, 20 200 ng of genomic DNA was used. PCR was performed 221 with the following thermal profile: initial denaturation at $94^{\circ} \mathrm{C}$ for $5 \mathrm{~min}$, followed by 34 cycles 222 of denaturation at $94^{\circ} \mathrm{C}$ for $30 \mathrm{~s}, 55^{\circ} \mathrm{C}$ for $30 \mathrm{~s}$, and extension at $72{ }^{\circ} \mathrm{C}$ for $30 \mathrm{~s}$, and a final 223 extension at $72^{\circ} \mathrm{C}$ for 10 min. The PCR products were separated in $3 \%$ agarose gels and then 224 visualized by ethidium bromide staining.

\section{Genotyping diverse rice varieties with allele-specific markers}

226 Genomic DNA of the 531 varieties was subjected to genotyping the presence of Pi-1, Pik- $p$, and 227 K-type alleles using the Pil-In and Pikp-Del markers. In addition, two previously reported 228 dCAPS markers Pi1FNP and dCAPS-795 (Hua et al. 2012; Zhai et al. 2011) which targeted the 229 functional regions of $P i-1$ and $P i k-p$, respectively, were used for verification. For genotyping 230 using the PilFNP marker, PCR amplicons were digested with RsaI. For genotyping using the 231 dCAPS-1879 marker, PCR amplicons were digested with Nde I. All the above products were 232 separated electrophoretically in 3\% agarose gels and then visualized by ethidium bromide 233 staining. 
237 We thank Dr. Qinghua Pan (Laboratory of Plant Resistance and Genetics, South China 238 Agricultural University) and Dr. Guo-Liang Wang (Department of Plant Pathology, Ohio State 239 University) for Magnaporthe oryzae isolates assistance. The research was funded by the Youngth 240 Program of Fujian Academy of Agricultural Sciences (YC2019004), the China Postdocotral 241 Science Foundation(2019M662219), the Projection of Public Welfare of Fujian Province 242 (2017R1019-10), and National Natural Science Foundation of China (31640006).

\section{Abbreviations}

244 InDel insertion/deletion

245 MAS marker-assisted selection

246 PCR polymerase chain reaction

247 SSR simple sequence repeat

248 dCAPS derived cleaved amplified polymorphic sequences

249 CTAB Cetyltrimethyl Ammonium Bromide

250 Authors' contributions

251 DT and ZW conceived and designed the experiments. DT, ZQ C, ZJ C, JL and YL conducted 252 experiment and analyzed the data. DT wrote and PJ, MW, S Chen, FW and ZW revised the 253 manuscript. All authors read and approved the final manuscript.

\section{Funding}

255 The Youngth Program of Fujian Academy of Agricultural Sciences (YC2019004), the China 
256 Postdocotral Science Foundation(2019M662219), the Projection of Public Welfare of Fujian

257 Province (2017R1019-10), and National Natural Science Foundation of China (31640006).

\section{Availability of data and materials}

259 The datasets supporting the conclusions of this article are included within the article and its

260 additional files.

261 Ethics approval and consent to participate

262 Not applicable.

263 Consent for publication

264 Not applicable.

265 Competing interests

266 The authors declare no conflicts of interest.

267 Additional files

268 Additional file 1: Table S1. List of rice varieties assessed in this study

269 Additional file 2:Table S2. Summary of identification results with Pi1-In and Pikp-Del markers

270 in 531 rice varieties

271 Authors'Affiliations

$272{ }^{1}$ Biotechnology Research Institute, Fujian Key Laboratory of Genetic Engineering for 
$274{ }^{2}$ State Key Laboratory of Ecological Pest Control for Fujian and Taiwan Crops, College of Life 275 Science, Fujian Agriculture and Forestry University, Fuzhou, China;

$276{ }^{3}$ Marine and Agricultural Biotechnology Laboratory, Institute of Oceanography, Minjiang 277 University, Fuzhou 350108, China;

$278{ }^{4}$ College of Biology and the Environment, Nanjing Forestry University, Nanjing, 210037, China;

$279{ }^{5}$ Department of Plant Pathology, University of Georgia, Tifton, GA 31793, USA.

\section{References}

283

Ashikawa I, Hayashi N, Yamane H, Kanamori H, Wu J, Matsumoto T, Ono K, and Yano M (2008) Two adjacent nucleotide- binding site-leucine-rich repeat class genes are required to confer Pikm-specific rice blast resistance. Genetics 180 (4):2267-2276. doi.org/10.1534/genetics. 108.095034 .

Brar D, Khush G (1997) Alien introgression in rice. Plant Mol. Biol. 35:35-47. doi.org/10.1007/ 978-94-011-5794-0_4.

Campbe MA, Chen D, Ronald PC (2004) Development of co-dominant amplified polymorphic sequence markers in rice that flank the Magnaporthe grisea resistance gene Pi7(t) in recombinant inbred line 29. Phytopathology. 94 (3):302-307. doi.org/10.1094/PHYTO. 2004.94.3.302.

Chen Z, Tian D, Lian T, Chen Z, Hu C, Wang F, and Chen S (2016) Characterization of the 
genotypes at the rice blast resistance Pik locus in 229 rice cultivars and important breeding materials. Fujian J. Agric. Sci. 31(6):553-559.(In Chinese).

Deng Y, Zhai K, Xie Z, Yang DY, Zhu XD, Liu JZ, Wang X, Qin P, Yang YZ, Zhang GM, Li Q, Zhang JF, Wu SQ, Milazzo JI, Mao BZ, Wang ET, Xie HA, Tharreau D, and He ZH (2017) Epigenetic regulation of antagonistic receptors confers rice blast resistance with yield balance. Science 355:962-965. doi:10.1126/science.aai8898.

Hayashi K, Yasuda N, Fujita Y, Koizumi S, and Yoshida H (2010) Identification of the blast resistance gene pit in rice cultivars using functional markers. Theor. Appl. Genet. 121(7):1357-1367.doi: 10.1007/s00122-010-1393-7.

He C, Chen T, Zhang Y.D, Zhu Z, Zhao QY, Zhou LH, Yu X, and Wang CL (2014) Genotypic analysis of blast resistance genes $P i$-ta and $P i-b$ for Japonica rice varieties and lines in Jiangsu Province. Jiangsu Agric. Sci. 30(5):921-927.(In Chinese).

$\mathrm{Hu} \mathrm{J}$, Li X, and Wu C (2010) Gene pyramiding to improve the resistance of rice hybrids to brown plant hopper and blast disease using molecular marker-assisted selection. Mol. Plant Breed. 8:1180-1187.(In Chinese).

Hua L, Wang W, Chen S, Wang C, Zeng L, Yang J, Zhu X, Su J (2015) Development of specific DNA markers for detecting the rice blast resistance gene alleles Pi2/9/z-t (In Chinese with English summary). Chin J Rice Sci 29:305-310. doi:10.3969/j.issn.1001-7216.2015.03.010.

Hua L, Wu J, Chen C, Wu W, He X, Lin F, Wang L, Ashikawa I, Matsumoto T, Wang L, Pan Q: The isolation of Pil, an allele at the Pik locus which confers broad spectrum resistance to rice blast. Theor Appl Genet 2012,125(5):1047-1055. doi:10.1007/s00122-012-1894-7.

Jeung JU, Kim BR, Cho YC, Han SS, Moon HP, Lee YT, et al. A novel gene, Pi40(t), linked to the DNA markers derived from NBS-LRR motifs confers broad spectrum of blast resistance 
in rice. Theor Appl Genet. 2007;115:1163-77. doi.org/10.1007/s00122-007-0642-x.

318 Jiang H, Feng Y, Bao L, Li X, Gao G, Zhang Q, Xiao J, Xu C, He Y (2012a) Improving blast 319 resistance of Jin 23B and its hybrid rice by marker-assisted gene pyramiding. Mol Breed 30:1679-1688. doi:10.1007/s11032-012-9751-6.

Khush GS (2005) What it will take to feed 5.0 billion rice consumers in 2030. Plant Mol Biol 59:1-6.doi:10.1007/s11103-005-2159-5.

Kiyosawa S (1987) With genetic view on the mechanism of resistance and virulence. Jpn J Iden 41:89-92 (In Japanese).

Kumari A, Das A, Devanna B, Thakur S, Singh P, Singh N, Sharma T (2013) Mining of rice blast resistance gene Pi54 shows effect of single nucleotide polymorphisms on phenotypic expression of the alleles. Eur J Plant Pathology 137:55-65. doi: 10.1007/s10658-013 $-0216-5$.

Li J, Li C, Chen Y, Lei C, Ling Z (2005) Evaluation of twenty-two blast resistance genes in Yunnan using monogenetic rice lines. Acta Phytophylacica Sin 32:113-119 (In Chinese).

Li CG, Wang D, Peng SS, Chen Y, Su P, Chen JB, Zheng LM, Tan XQ, Liu JL, Xiao YH, Kang HX, Zhang DY(2019) Genome-wide association mapping of resistance against rice blast strains in South China and identification of a new Pik allele. Rice 12:47. doi:10.1186/s 12284-019-0309-7

Ma J, Lei C, Xu X et al (2015) Pi64, encoding a novel CC-NBS-LRR Protein, confers resistance to leaf and neck blast in rice. Mol Plant-Microbe Interact 28:558-568. doi: 10.1094/ MPMI-11-14-0367-R

Murray MG, Thompson WF (1980) Rapid isolation of high molecular weight plant DNA. Nucleic Acids Res 8:4321-4325. doi:10.1093/nar/8.19.4321 
Nawaz MA, Rehman HM, Baloch FS, Ijaz B, Ali MA, Khan IA, Lee JD, Chung G, Yang SH(2017) Genome and transcriptome-wide analysis of cellulose synthase gene superfamily in soybean. J. Plant Physiol. 215:163-175. doi:10.1016/j.jplph.2017.04.009

Pan QH, Hu Z, Tanisaka T, Wang L (2003) Fine mapping of the blast resistance gene Pi15, linked to Pii, on rice chromosome 9. Acta Bot Sin 45:871-877(In Chinese).

Ramkumar G, Srinivasarao K, Madhan Mohan K, Sudarshan I, Sivaranjani AKP, Gopalakrishna K, Neeraja CN, Balachandran SM, Sundaram RM, Prasad MS, Shobha Rani N, Rama Prasad AM, Viraktamath BC, Madhav MS (2011) Development and validation of functional marker targeting an InDel in the major rice blast disease resistance gene Pi54(Pikh). Mol Breed 27:129-135. doi:10.1007/s11032-010-9538-6

RoyChowdhury M, Jia Y, Jackson A, Jia MH, Fjellstrom R, Cartwright RD (2012) Analysis of rice blast resistance gene $P i-z$ in rice germplasm using pathogenicity assays and DNA markers. Euphytica 184:35-46. doi.org/10.1007/s10681-011-0481-3.

Shomura A, Izawa T, Ebana K, Ebitani T, Kanegae H, Konishi S, Yano M (2008) Deletion in a gene associated with grain size increased yields during rice domestication. Nat Genet 40:1023-1028. doi:10.1038/ng.169.

Tian DG, Chen ZJ, Chen Z Q, Zhou YC, Wang ZH, Wang F, Chen SB (2016) Allele-specific marker-based assessment revealed that the rice blast resistance genes $P i 2$ and $P i 9$ have not been widely deployed in Chinese indica rice cultivars. Rice 9:19. doi:10.1186/s12284-016-0091-8.

Tsunematsu H, Yanoria MJT, Ebron LA, Hayashi N, Ando I, Kato H, Imbe T, Khush GS (2000) Development of monogenic lines for rice blast resistance. Breed Sci 50:229-234. doi:10.1270/jsbbs.50.229. 
Wang B, Ebbole DJ, Wang Z (2017) The arms race between Magnaporthe oryzae and rice: diversity and interaction of Avr and R genes. J Integr Agric 16:2746-2760. doi: https://doi.org/10.1016/S2095-3119(17)61746-5.

Wang FQ, Chen ZH, Xu Y, Wang J, Li WQ, Fan FJ, Chen LQ, Tao YJ, Zhong WG, and Yang J (2019) Development and application of the functional marker for the broad-spectrum blast resistance gene PigmR in rice. Scientia Agric. Sin. 52(6):955-967.(In Chinese).

Wang L, Xu XK, Lin F, Pan QH (2009) Characterization of rice blast resistance genes in the Pik cluster and fine mapping of the Pik-p locus. Phytopathology 99:900-905. doi:10.1094/PHYTO-99-8-0900.

Wang WJ, Zhou JY, Wang CY, Su J, Feng JQ, Chen B, Feng AQ, Yang JY, Chen S, Zhu XY(2017) Distribution of eight rice blast resistance genes in indica hybrid rice in China. Chin J Rice Sci, 2017, 31(3): 299-306. (In Chinese).

Xu Y, McCouch SR, Zhang Q (2005) How can we use genomics to improve cereals with rice as a reference genome? Plant Mol Biol 59:7-26. doi:10.1007/s11103-004-4681-2.

Yang X, Zhu C, Ruan H, Du Y, Guan R, Chen F (2008) Pathogenic types of Magnaporthe grisea Barr. and resistance of some rice cultivars to the pathogens in Fujian province. $\mathrm{J}$ Fujian Agr Fore Uni 37:243-247 (In Chinese)

Yuan B, Zhai C, Wang W, Zeng X, Xu X, Hu H, Lin F, Wang L, Pan Q (2011) The Pik-presistance to Magnaporthe oryzae in rice is mediated by a pair of closely linked CC-NBS-LRR genes. Theor Appl Genet 122:1017-1028. doi:10.1007/s00122-010- 1506-3

Zeigler RS, Tohme J, Nelson R, Levy M, Correa-Victoria FJ (1994) Lineage exclusion: a proposal for linking blast population analysis to resistance breeding. In: Zeigler RS, Leong SA, Teng PS (eds) Rice blast disease. CAB International, Wallingford, pp 267-292 
386 Zhai C, Lin F, Dong Z, He X, Yuan B, Zeng X, Wang L, Pan Q (2011) The isolation and 387 characterization of Pik, a rice blast resistance gene which emerged after rice domestication. $388 \quad$ New Phytol 189:321-334. doi:10.1111/j.1469-8137.2010.03462.x

389 Zhai C, Zhang Y, Yao N, Lin F, Liu Z, Dong Z, Wang L, Pan Q (2014)Function and interaction of 390 the coupled genes responsible for $P i k-h$ encoded rice blast resistance. PLoS One 391 9(6):e98067. doi:10.1371/journal.pone.0098067

392 Zhang C, Ma J, Xiao J, Liu Y, Xin A, Ren Y (2010) The blast resistance of 24 monogenic rice 393 lines to prevalence physiologic races of Heilongjiang and analysis of pathogenicity $394 \quad$ association. Chi Agr Sci Bull 26:233-237 (In Chinese).

395 
Table 1. List of primers in the present study

\begin{tabular}{|c|c|c|c|}
\hline Primers & Sequence: $5^{\prime}-3^{\prime}$ & Expected size (bp) & Comments \\
\hline PikpDel-F & TGGTTAAATAGGACTCCCTC & $\begin{array}{l}368 \text { for } P i k-p \text { allele, } \\
377 \text { for other functional alleles } \\
\text { of } P i k \text { locus }\end{array}$ & $\begin{array}{l}\text { Forward primer to amplify the } \\
\text { fragments corresponding to the } \\
\text { Pik locus region for allele mining }\end{array}$ \\
\hline PikpDel-R & CATTCGCAGACTCGTTGA & $\begin{array}{l}368 \text { for } P i k-p \text { allele, } \\
377 \text { for other functional alleles } \\
\text { of } P i k \text { locus }\end{array}$ & $\begin{array}{l}\text { Reverse primer to amplify the } \\
\text { fragments corresponding to the } \\
\text { Pik locus region for allele mining }\end{array}$ \\
\hline Pi1In-F & TGAGGTAGAAGCGGGAGC & $\begin{array}{l}218 \text { for } P i-1 \text { allele, } \\
207 \text { for other functional alleles } \\
\text { of } P i k \text { locus }\end{array}$ & $\begin{array}{l}\text { Forward primer to amplify the } \\
\text { fragments corresponding to the } \\
\text { Pik locus region for allele mining }\end{array}$ \\
\hline Pi1In-R & GGTTGGTCGAAACCAGAA & $\begin{array}{l}218 \text { for } P i-1 \text { allele, } \\
207 \text { for other functional alleles } \\
\text { of } P i k \text { locus }\end{array}$ & $\begin{array}{l}\text { Reverse primer to amplify the } \\
\text { fragments corresponding to the } \\
\text { Pik locus region for allele mining }\end{array}$ \\
\hline Pi1FNP-F & CAATAGTCCAGCTAAAACGG & $\begin{array}{l}159 \text { for } P i-1 \text { allele, } \\
178 \text { for non- } P i-1 \text { alleles }\end{array}$ & $\begin{array}{l}\text { Forward primer for allele mining } \\
\text { between } P i 1 \text { and non-Pil alleles, } \\
\text { and for dCAPS marker Pi1FNP }\end{array}$ \\
\hline PilFNP-R & CATTGCGCCTTTACCTTGT & $\begin{array}{l}159 \text { for } P i-1 \text { allele, } \\
178 \text { for non- } P i-1 \text { alleles }\end{array}$ & $\begin{array}{l}\text { Forward primer for allele mining } \\
\text { between } P i 1 \text { and non-Pil alleles, } \\
\text { and for dCAPS marker Pi1FNP }\end{array}$ \\
\hline dCAPS-1879-F & CGTGGAAGTTCAACAAAAGG & $\begin{array}{l}73 \text { for } \text { Pik- } p \text { allele, } \\
115 \text { for non-Pik- } p \text { alleles }\end{array}$ & $\begin{array}{l}\text { Forward primer for allele mining } \\
\text { between } P i k-p \text { and non-Pikp } \\
\text { alleles, and for dCAPS marker } \\
\text { dCAPS-1879 }\end{array}$ \\
\hline dCAPS-1879-R & CAGCACCTGTATTATCCCAT & $\begin{array}{l}73 \text { for } \text { Pik- } p \text { allele, } \\
115 \text { for non-Pik- } p \text { alleles }\end{array}$ & $\begin{array}{l}\text { Forward primer for allele mining } \\
\text { between } P i k-p \text { and non-Pikp } \\
\text { alleles, and for dCAPS marker } \\
\text { dCAPS-1879 }\end{array}$ \\
\hline
\end{tabular}


Table 2. Disease reactions of representative varieties $P i-1, P i k-p$, K-type-contained using diverse

\section{Magnaporthe grisea strains}

\begin{tabular}{|c|c|c|c|c|c|c|c|c|}
\hline Genes & Cultivars & KJ201 & CHNOS60-2-3 & CHE86061 & FJ501-3 & FJ86 & CHL2110 & CHL768 \\
\hline \multirow{8}{*}{$P i-1$} & Enhui 99-64 & 0 & 0 & 0 & 0 & 0 & 0 & 0 \\
\hline & IR0437 & 0 & 0 & 2 & 0 & 0 & 0 & 0 \\
\hline & IR56455-206-2-1-2R & 0 & 0 & 1 & 0 & 1 & 2 & 0 \\
\hline & IR0436 & 0 & 0 & 0 & 1 & 0 & 0 & 0 \\
\hline & Chenghui 448 & 0 & 0 & 0 & 0 & 0 & 0 & 0 \\
\hline & RGD-7S & 0 & 0 & 3 & 0 & 0 & 0 & 0 \\
\hline & Jinkang1B & 0 & 0 & 1 & 0 & 0 & 0 & 0 \\
\hline & BL123 & 0 & 0 & 0 & 0 & 0 & 0 & 0 \\
\hline \multirow{4}{*}{ Pik-p } & RCPL3-2 & 0 & 0 & 2 & 0 & 0 & 0 & 0 \\
\hline & Ruanmigu & 0 & 0 & 0 & 0 & 0 & 0 & 0 \\
\hline & Zaohui 62 & 0 & 0 & 0 & 0 & 0 & 0 & 0 \\
\hline & Huifeng A & 0 & 0 & 0 & 0 & 0 & 0 & 0 \\
\hline \multirow{6}{*}{ K-type } & Gufeng A & 0 & 0 & 0 & 0 & 0 & 4 & 0 \\
\hline & Minhui3301 & 0 & 0 & 0 & 5 & 0 & 0 & 0 \\
\hline & Shuiyuan 290 & 0 & 0 & 0 & 5 & 0 & 0 & 0 \\
\hline & IR04557 & 0 & 0 & 0 & 5 & 0 & 0 & 0 \\
\hline & IR73712-68-3-1-2 & 0 & 0 & 0 & 5 & 0 & 0 & 0 \\
\hline & GIZA176 & 0 & 0 & 0 & 5 & 5 & 0 & 0 \\
\hline \multirow{4}{*}{$\begin{array}{c}\text { Monogenic } \\
\text { lines }\end{array}$} & IRBLkp-K60(Pik-p) & 1 & 0 & 5 & 0 & 0 & 0 & 0 \\
\hline & F-98-7 (Pik-m) & 0 & 0 & 5 & 0 & 0 & 0 & 0 \\
\hline & IRBL1-CL(Pi-l) & 0 & 0 & 4 & 0 & 1 & 4 & 0 \\
\hline & IRBLkh-K3(Pik-h) & 1 & 0 & 5 & 1 & 0 & 0 & 0 \\
\hline \multirow{2}{*}{$\begin{array}{c}\text { Negative } \\
\text { (CK) }\end{array}$} & Nipponbare & 3 & 5 & 2 & 5 & 5 & 3 & 5 \\
\hline & Co39 & 5 & 5 & 2 & 5 & 5 & 4 & 5 \\
\hline
\end{tabular}


Fig.1 Sequence alignment showing the 19-bp and 11-bp InDel in the Pik-p-1-1015-bp and Pi1-5+6816-bp alleles, respectively. (A) Physical location of the identified InDel region in the Pik locus. (B) and (C) Sequence alignment showing the 19-bp InDel between the Pik-p and non-Pik-p alleles and the 11-bp InDel between the Pi-1 and non-Pi-1 alleles in IRBL1-CL-Pi1( Pi-1), IRBLkp-K60-Pik-p (Pik-p), IRBLk-Ka-Pik (Pi-k), IRBLkm-Ts-Pik-m (Pik-m), IRBLkh-K3-Pik-h (Pik-h), and IRBLks-S-Pik-s (Pik-s). (D) PCR amplified with markers Pi1-In and Pikp-Del, respectively, derived from IRBL1-CL-Pi1(Pi-1), IRBLkp-K60-Pik-p (Pik-p), IRBLk-Ka-Pik (Pi-k), IRBLkm-Ts-Pik-m (Pik-m), IRBLkh-K3-Pik-h (Pik-h), IRBLks-S-Pik-s (Pik-s), and NPB were used for electrophoresis analysis.

Fig. 2 Electrophoresis profiles of the Pikp-Del and Pi1-In marker that differentiates the Pik-p and Pi-1 alleles. (A) Pikp-Del and dCAPS -1879 marker that differentiates the Pik-p and non-Pik-p alleles. Upper panel showing the PCR products with Pikp-Del and lower panel showing the dCAPS -1879 PCR products digested with NdeI. (B) Pikp-Del, Ku1-GT, and Ku2-GT marker that differentiates the $\mathrm{K}$ genotype and non-K genotype alleles. Upper panel showing the PCR products with Pikp-Del, middle panel showing PCR products with Kul-GT, and lower panel showing PCR products with Ku2-GT. (C) Pi1-In and Pi1FNP marker that differentiates the Pi-1 and non-Pi-1 alleles. Upper panel showing the PCR products with Pil-In and lower panel showing the PCR products of Pi1FNP digested with Rsa I.

Fig. 3 Co-segregation analysis on Pikp-Del and Pi1-In with blast resistance using rice varieties containing Pik-p and Pi-1. A total of 8 varieties containing Pi-1, 4 containing Pik-p and 3 
426 isogenic lines as check varieties were used for inoculation identification by Magnaporthe oryzae 427 isolates CHL768 (contain-AvrPi-1) and CHL2110 (contian-AvrPik-p), respectively. 


\section{Figures}

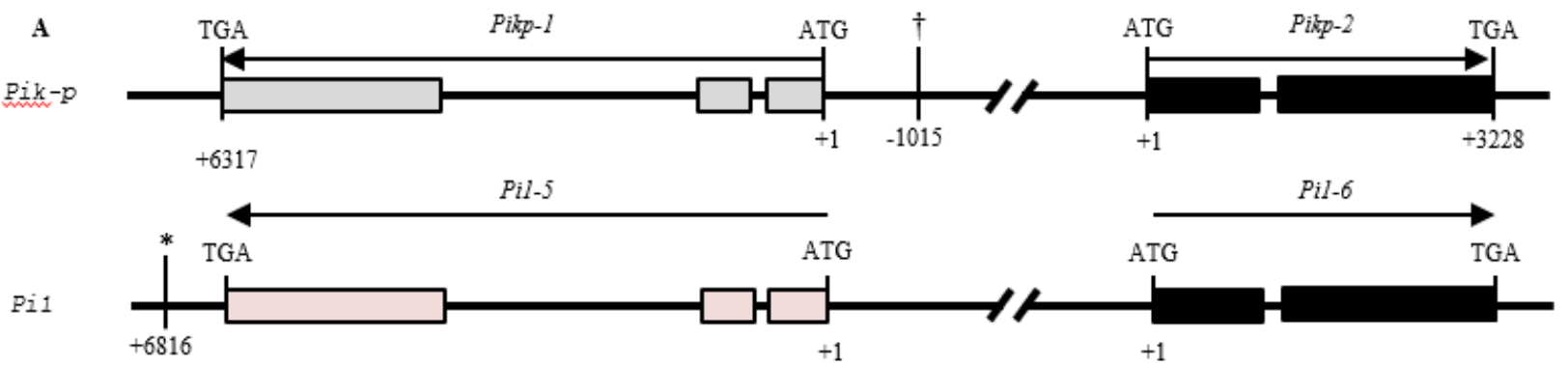

B

Plkp.txt

Pl1.txt

GACCTCT TT TT TATT AAATT AT TATT.

CATGCT CT CT GAT TCTATT GGATGCATGCAT TGTATT TATT AGGAT GT TCT AAAC GACCTCT TT TT TATT AAATT AT TATTT TT TAACAT CCT CT ACACTCATGTT CT CT GAT TCTATT GGATGCATGCAT TGTATT TATT AGGAT GT TCT AAAC

Plk.txt

Plkh.txt

Prkm.txt

GACC TCT TT TT TATT AAATT AT TATT TT TAACAT CCT CT ACAC TCATGTT CT CT GAT TCTATT GGATGCATGCAT TGTATT TATT AGGAT GT TCT AAAC GACCTCT TT TT TATT AAATT AT TATTT TT TAACAT CCT CT AC AC TCATGTT CT CT GAT TCTATT GGATGCATGCAT TGTATT TATT AGGAT GT TCT AAAC

GACC TCT TT TT TATT AAATT AT TATT TT TAACAT CCT CT ACAC TCATGTT CT CT GAT TCTATT GGATGCATGCAT TGTATT TATT AGGAT GT TCTAAAC

GACCTCT TT TT TATT AAATT AT TATT TT TAACAT CC T CT ACAC TCATGTTCT CT GAT TCTATT GGATGCATGCAT TGTATT TATT AGGAT GT TCT AAAC

C

Pi1.txt

Pikp.txt

Pik.txt

Pikh.txt

Pi-km.txt

Piks.txt

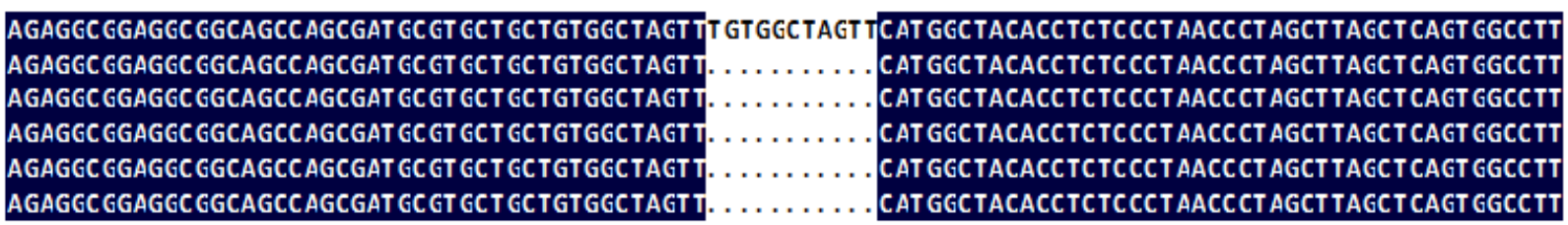

D
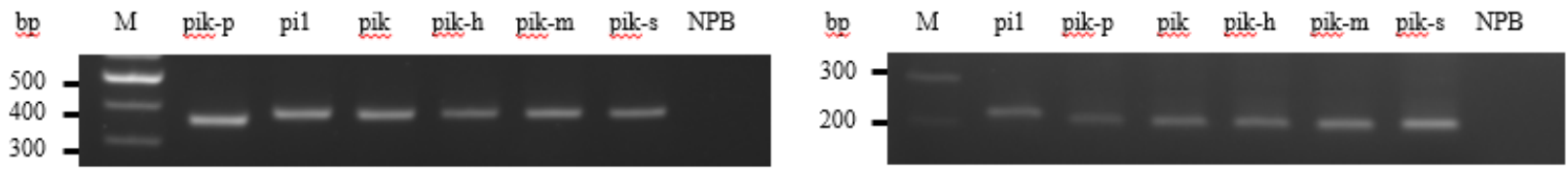

Figure 1

Sequence alignment showing the 19-bp and 11-bp InDel in the Pik-p-1-1015-bp and Pi1-5+6816-bp alleles, respectively. (A) Physical location of the identified InDel region in the Pik locus. (B) and (C) Sequence alignment showing the 19-bp InDel between the Pik-p and non-Pik-p alleles and the 11-bp InDel between the Pi-1 and non-Pi-1 alleles in IRBL1-CL-Pi1 ( Pi-1), IRBLkp-K60-Pik-p (Pik-p), IRBLk-Ka-Pik (Pi-k), IRBLkmTs-Pik-m (Pik-m), IRBLkh-K3-Pik-h (Pik-h), and IRBLks-S-Pik-s (Pik-s). (D) PCR amplified with markers Pi1In and Pikp-Del, respectively, derived from IRBL1-CL-Pi1(Pi-1), IRBLkp-K60-Pik-p (Pik-p), IRBLk-Ka-Pik (Pik), IRBLkm-Ts-Pik-m (Pik-m), IRBLkh-K3-Pik-h (Pik-h), IRBLks-S-Pik-s (Pik-s), and NPB were used for electrophoresis analysis. 


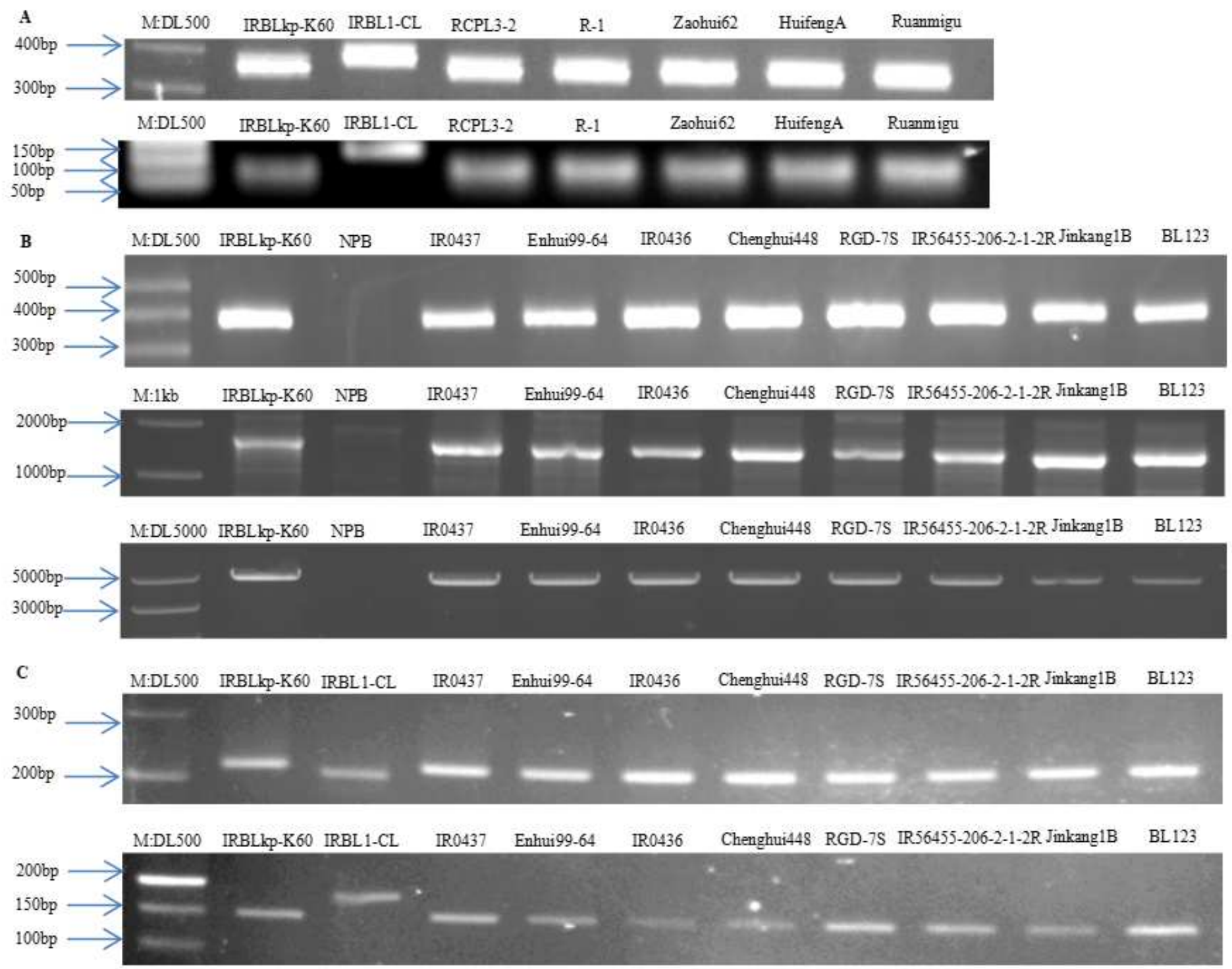

\section{Figure 2}

Electrophoresis profiles of the Pikp-Del and Pi1-In marker that differentiates the Pik-p and Pi-1 alleles. (A) Pikp-Del and dCAPS -1879 marker that differentiates the Pik-p and non-Pik-p alleles. Upper panel showing the PCR products with Pikp-Del and lower panel showing the dCAPS -1879 PCR products digested with Ndel. (B) Pikp-Del, Ku1-GT, and Ku2-GT marker that differentiates the K genotype and non-K genotype alleles. Upper panel showing the PCR products with Pikp-Del, middle panel showing PCR products with Kul-GT, and lower panel showing PCR products with Ku2-GT. (C) Pi1-In and Pi1FNP marker that differentiates the Pi-1 and non-Pi-1 alleles. Upper panel showing the PCR products with Pi1-In and lower panel showing the PCR products of Pi1FNP digested with Rsa I. 


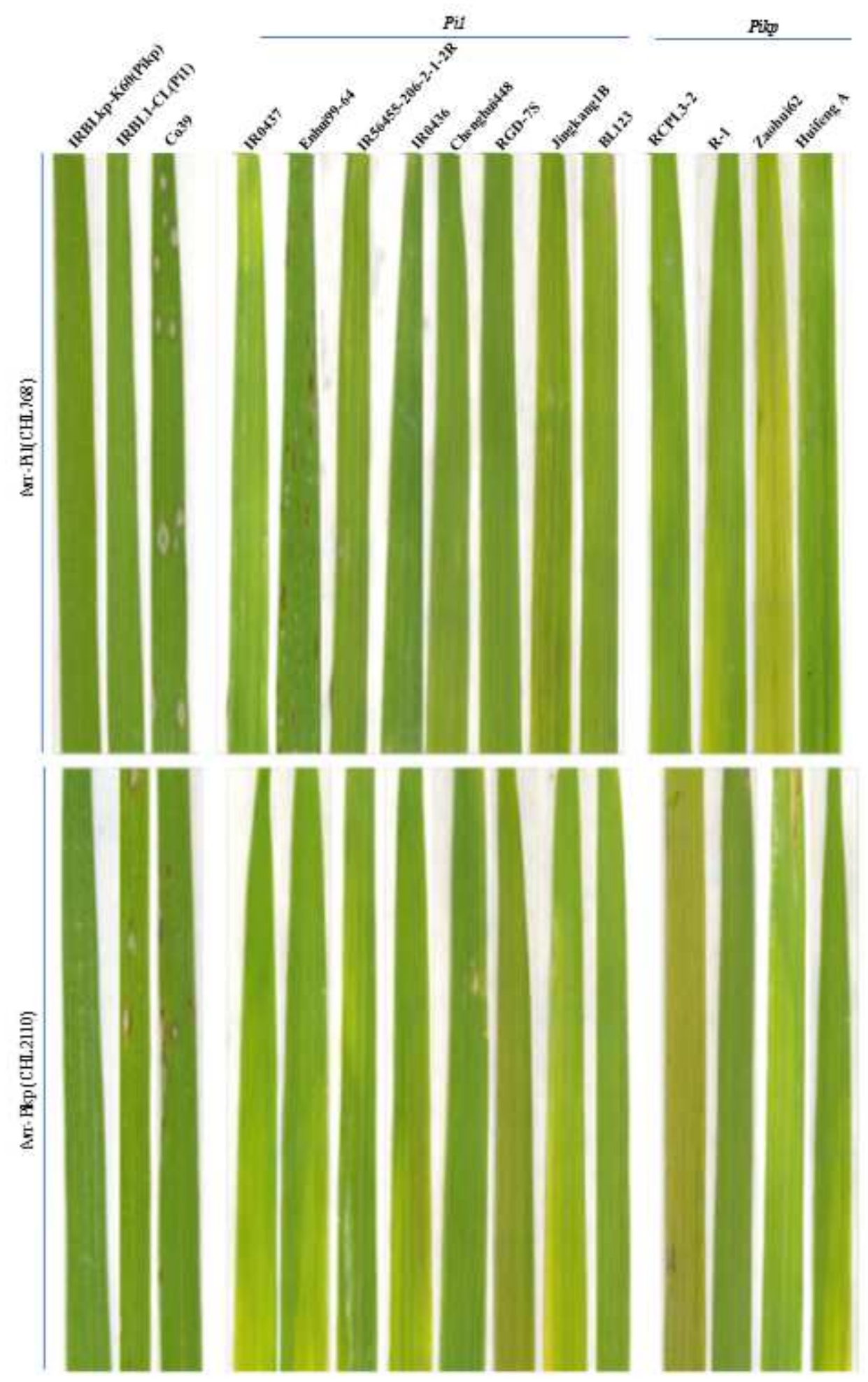

Figure 3

Co-segregation analysis on Pikp-Del and Pi1-In with blast resistance using rice varieties containing Pik-p and Pi-1. A total of 8 varieties containing Pi-1, 4 containing Pik-p and 3 isogenic lines as check varieties were used for inoculation identification by Magnaporthe oryzae isolates CHL768 (contain-AvrPi-1) and CHL2110 (contian-AvrPik-p), respectively.

\section{Supplementary Files}


This is a list of supplementary files associated with this preprint. Click to download.

- AdditionalTableS2.docx

- AdditionalTables1Ricevarietiesassessedinthisstudy.docx 